\title{
Vibration and two-photon absorption
}

\author{
David M. Bishop ${ }^{\text {a) }}$ and Josep M. Luis ${ }^{\text {b) }}$ \\ Department of Chemistry, University of Ottawa, Ottawa K1N 6N5, Canada \\ Bernard Kirtman \\ Department of Chemistry and Biochemistry, University of California, Santa Barbara, California 93106
}

(Received 13 December 2001; accepted 20 March 2002)

A treatment of nuclear vibrational motions in two-photon absorption is laid out in detail. Perturbation theory is used to develop working formulas for diatomic molecules and tested for the $X^{1} \Sigma^{+}-A{ }^{1} \Pi$ transition of carbon monoxide. The results are compared with the exact numerical values. Certain vibrational contributions, not previously recognized, are found to make an important contribution to the two-photon absorption probability. (C) 2002 American Institute of Physics.

[DOI: $10.1063 / 1.1477179]$

\section{INTRODUCTION}

The point of this paper is to investigate in a rigorous and transparent fashion the role of nuclear vibrational motions in two-photon absorption (TPA). The over-riding spirit is the same as that which was successfully used to ascertain vibrational effects in the evaluation of the hyperpolarizabilities that govern nonlinear optical (NLO) processes in the nonresonant regime. ${ }^{1-5}$ Interest in TPA arises because molecules, which in the presence of intense laser beams can absorb two photons simultaneously with a large absorption cross section, can be used in various practical ways. ${ }^{6}$ These include twophoton excited fluorescence, ${ }^{7}$ optical power limiting, ${ }^{8,9}$ three-dimensional optical data storage, ${ }^{10}$ two-photon induced biological caging experiments, ${ }^{11}$ up-converting lasing, early corrosion detection, and photodynamic therapy. ${ }^{12,13}$ Another quite different reason for interest is that in NLO processes the measure of efficacy is the figure of merit ${ }^{14}$ and this involves both linear and nonlinear absorption of the optical beam. A recent study of the linear absorption has been carried out by Norman et al. ${ }^{15}$

There have been many theoretical investigations of the purely electronic aspects of TPA and examples are Refs. 6, 16-20. The first theoretical treatment of vibronic coupling, based on the linear Herzberg-Teller term, was carried out by Honig and co-workers ${ }^{21,22}$ for benzene. Ab initio calculations were later carried out by Luo and co-workers, ${ }^{23,24}$ and the theory was extended to include the Franck-Condon distribution by Macak et al. ${ }^{25,26}$ Other related studies are by Sundholm et al. ${ }^{27}$ where vibrationally averaged transition moments were employed for $\mathrm{CO}$, and by Painelli et al. ${ }^{28}$ who used an approximate two-state model to account for electronic-vibrational coupling in push-pull chromophores. Here we present a more complete and more general approach to the vibrational problem.

\footnotetext{
${ }^{a}$ Electronic mail: dbishop@ @science.uottawa.ca

${ }^{b}$ Permanent address: Institute of Computational Chemistry and Department of Chemistry, University of Girona, Campus de Montilivi, 17071 Girona, Catalonia, Spain.
}

Basically, TPA cross-sections are dependent on the imaginary part of the second hyperpolarizability for the intensity-dependent refractive index (IDRI), when a twophoton frequency $(2 \omega)$ coincides or nearly coincides with a transition from a vibronic ground state to some vibronic excited state(s). In this perspective the general formulas for vibrational effects in TPA can be found by using the BishopKirtman (BK) approach to vibrational hyperpolarizabilities. ${ }^{1-3}$ As a specific application of the method developed here, we have considered carbon monoxide. By taking a diatomic molecule we are able to carry out and compare two different ways of evaluating the required transition moments: numerical integration (with essentially exact vibrational wavefunctions ${ }^{29}$ ) and perturbation theory incorporating both electrical and mechanical anharmonicities. We determine the potential energy surface (PES) of the $X^{1} \Sigma^{+}$ ground and $A^{1} \Pi$ excited states of $\mathrm{CO}$ by means of the multiconfigurational self consistent field (MCSCF) method. Since in previous studies of polyatomic molecules it is often assumed that the PES of the ground and excited states are identical (see, e.g., Ref. 25) with the latter just shifted to a new equilibrium geometry and its corresponding energy, we also test this assumption here using $\mathrm{CO}$ as a model. Our aim here is not to provide definitive results for $\mathrm{CO}$ but rather to use this simple diatomic as the vehicle for evaluating the various aspects of our theoretical development, which will later be extended to polyatomic molecules.

Our primary results are (1) the perturbation treatment presented appears to be a viable approach for calculating the different vibrational contributions to TPA; (2) in total, these vibrational contributions can be important; and (3) attention should be directed towards the "pure" vibrational terms, which turn out to be particularly important for CO. In the next section we derive the general theory. This is followed by an outline of the application of perturbation theory for the diatomic case. Then comes a section on computational details, and finally we give our results and a discussion thereof. 


\section{GENERAL THEORY}

Our goal is the determination of two-photon absorption probabilities and cross sections. These are related to the imaginary parts of the IDRI hyperpolarizability tensor components. We therefore start with the general formula for any second-order electronic hyperpolarizability component $\gamma_{\alpha \beta \gamma \delta}$ as given by Orr and Ward: ${ }^{30}$

$$
\begin{aligned}
& \gamma_{\alpha \beta \gamma \delta}\left(-\omega_{\sigma} ; \omega_{1}, \omega_{2}, \omega_{3}\right)=\hbar^{-3} \sum P_{1,2,3}\left(\sum _ { K } { } ^ { \prime } \sum _ { L } { } ^ { \prime } \sum _ { M } { } ^ { \prime } \left(\frac{\left\langle 0\left|\hat{\mu}_{\alpha}\right| K\right\rangle\left\langle K\left|\overline{\hat{\mu}_{\beta}}\right| L\right\rangle\left\langle L\left|\overline{\hat{\mu}_{\gamma}}\right| M\right\rangle\left\langle M\left|\hat{\mu}_{\delta}\right| 0\right\rangle}{\left(\omega_{K}-i \Gamma_{K} / 2-\omega_{\sigma}\right)\left(\omega_{L}-i \Gamma_{L} / 2-\omega_{2}-\omega_{3}\right)\left(\omega_{M}-i \Gamma_{M} / 2-\omega_{3}\right)}\right.\right. \\
& +\frac{\left\langle 0\left|\hat{\mu}_{\beta}\right| K\right\rangle\left\langle K\left|\hat{\mu}_{\alpha}\right| L\right\rangle\left\langle L\left|\hat{\mu}_{\gamma}\right| M\right\rangle\left\langle M\left|\hat{\mu}_{\delta}\right| 0\right\rangle}{\left(\omega_{K}+i \Gamma_{K} / 2+\omega_{1}\right)\left(\omega_{L}-i \Gamma_{L} / 2-\omega_{2}-\omega_{3}\right)\left(\omega_{M}-i \Gamma_{M} / 2-\omega_{3}\right)} \\
& +\frac{\left\langle 0\left|\hat{\mu}_{\beta}\right| K\right\rangle\left\langle K\left|\hat{\mu}_{\gamma}\right| L\right\rangle\left\langle L\left|\hat{\mu}_{\alpha}\right| M\right\rangle\left\langle M\left|\hat{\mu}_{\delta}\right| 0\right\rangle}{\left(\omega_{K}+i \Gamma_{K} / 2+\omega_{1}\right)\left(\omega_{L}+i \Gamma_{L} / 2+\omega_{1}+\omega_{2}\right)\left(\omega_{M}-i \Gamma_{M} / 2-\omega_{3}\right)} \\
& \left.+\frac{\left\langle 0\left|\hat{\mu}_{\beta}\right| K\right\rangle\left\langle K\left|\hat{\hat{\mu}}_{\gamma}\right| L\right\rangle\left\langle L\left|\overline{\hat{\mu}_{\delta}}\right| M\right\rangle\left\langle M\left|\hat{\mu}_{\alpha}\right| 0\right\rangle}{\left(\omega_{K}+i \Gamma_{K} / 2+\omega_{1}\right)\left(\omega_{L}+i \Gamma_{L} / 2+\omega_{1}+\omega_{2}\right)\left(\omega_{M}+i \Gamma_{M} / 2+\omega_{\sigma}\right)}\right) \\
& -\sum_{K}{ }^{\prime} \sum_{L}{ }^{\prime}\left(\frac{\left\langle 0\left|\hat{\mu}_{\alpha}\right| K\right\rangle\left\langle K\left|\hat{\mu}_{\beta}\right| 0\right\rangle\left\langle 0\left|\hat{\mu}_{\gamma}\right| L\right\rangle\left\langle L\left|\hat{\mu}_{\delta}\right| 0\right\rangle}{\left(\omega_{K}-i \Gamma_{K} / 2-\omega_{\sigma}\right)\left(\omega_{K}-i \Gamma_{K} / 2-\omega_{1}\right)\left(\omega_{L}-i \Gamma_{L} / 2-\omega_{3}\right)}\right. \\
& +\frac{\left\langle 0\left|\hat{\mu}_{\alpha}\right| K\right\rangle\left\langle K\left|\hat{\mu}_{\beta}\right| 0\right\rangle\left\langle 0\left|\hat{\mu}_{\gamma}\right| L\right\rangle\left\langle L\left|\hat{\mu}_{\delta}\right| 0\right\rangle}{\left(\omega_{K}-i \Gamma_{K} / 2-\omega_{1}\right)\left(\omega_{L}+i \Gamma_{L} / 2+\omega_{2}\right)\left(\omega_{L}-i \Gamma_{L} / 2-\omega_{3}\right)} \\
& +\frac{\left\langle 0\left|\hat{\mu}_{\beta}\right| K\right\rangle\left\langle K\left|\hat{\mu}_{\alpha}\right| 0\right\rangle\left\langle 0\left|\hat{\mu}_{\gamma}\right| L\right\rangle\left\langle L\left|\hat{\mu}_{\delta}\right| 0\right\rangle}{\left(\omega_{K}+i \Gamma_{K} / 2+\omega_{1}\right)\left(\omega_{K}+i \Gamma_{K} / 2+\omega_{\sigma}\right)\left(\omega_{L}+i \Gamma_{L} / 2+\omega_{2}\right)} \\
& \left.\left.+\frac{\left\langle 0\left|\hat{\mu}_{\beta}\right| K\right\rangle\left\langle K\left|\hat{\mu}_{\alpha}\right| 0\right\rangle\left\langle 0\left|\hat{\mu}_{\gamma}\right| L\right\rangle\left\langle L\left|\hat{\mu}_{\delta}\right| 0\right\rangle}{\left(\omega_{K}+i \Gamma_{K} / 2+\omega_{1}\right)\left(\omega_{L}+i \Gamma_{L} / 2+\omega_{2}\right)\left(\omega_{L}-i \Gamma_{L} / 2-\omega_{3}\right)}\right)\right) .
\end{aligned}
$$

This expression is valid provided all excited state populations remain negligible; it contains $\Gamma_{K}$ which is the population decay rate of the electronic excited stated $|K\rangle$. Without terms of the type $i \Gamma_{K} / 2$, one has the conventional expression for the second-order electronic hyperpolarizability components in the nonresonant region. However, the introduction of these phenomenological damping factors, ${ }^{31,32}$ which are related to the lifetime of the excited state $|K\rangle$ (dependent on spontaneous emissions and nonradiative processes), allows us to deal with the resonant and near-resonant region, where singularities would otherwise occur (such as in the case of TPA). $\gamma_{\alpha \beta \gamma \delta}$ is a complex quantity and its imaginary component is a measure of the absorption intensity for any second-order NLO process.

In this formula $\alpha, \beta, \gamma$ and $\delta$ refer to the molecular axes; $\omega_{1}, \omega_{2}$ and $\omega_{3}$ are optical frequencies and $\omega_{\sigma}$ is their sum; $\Sigma P_{1,2,3}$ indicates a sum over the terms obtained by the six permutations of the pairs $\left(\omega_{1} / \hat{\mu}_{\beta}\right),\left(\omega_{2} / \hat{\mu}_{\gamma}\right)$ and $\left(\omega_{3} / \hat{\mu}_{\delta}\right) ;|K\rangle$ is an electronic wavefunction with energy $\hbar \omega_{K}$ relative to the ground electronic state; $\hat{\mu}_{\mathrm{s}}=\hat{\mu}_{\mathrm{s}}-\left\langle 0\left|\hat{\mu}_{\mathrm{s}}\right| 0\right\rangle$ is the fluctuation dipole moment operator and $\hat{\mu}_{\mathrm{s}}$ is the dipole moment operator in the $\zeta$ Cartesian direction; the primes on the summation over the electronic states indicate exclusion of the ground state. The expression in Eq. (1) is necessarily a function of some arbitrarily chosen fixed nuclear geometry $(\mathbf{R})$.

As in the BK treatment of vibrational hyperpolarizabilities, ${ }^{1}$ we can now generalize the previous expression by replacing the electronic wavefunctions and energies by their vibronic counterparts and this leads to

$$
\begin{aligned}
\gamma_{\alpha \beta \gamma \delta}( & \left.-\omega_{\sigma} ; \omega_{1}, \omega_{2}, \omega_{3}\right) \\
= & \hbar^{-3} \sum P_{1,2,3}\left(\sum _ { k , K } { } ^ { \prime } \sum _ { l , L } { } ^ { \prime } \sum _ { m , M } { } ^ { \prime } \left(\frac{\left\langle 0,0\left|\hat{\mu}_{\alpha}\right| K, k\right\rangle\left\langle k, K\left|\overline{\hat{\mu}_{\beta}}\right| L, l\right\rangle\left\langle l, L\left|\overline{\hat{\mu}_{\gamma}}\right| M, m\right\rangle\left\langle m, M\left|\hat{\mu}_{\delta}\right| 0,0\right\rangle}{\left(\omega_{k K}-i \Gamma_{k K} / 2-\omega_{\sigma}\right)\left(\omega_{l L}-i \Gamma_{l L} / 2-\omega_{2}-\omega_{3}\right)\left(\omega_{m M}-i \Gamma_{m M} / 2-\omega_{3}\right)}\right.\right. \\
& +\frac{\left\langle 0,0\left|\hat{\mu}_{\beta}\right| K, k\right\rangle\left\langle k, K\left|\hat{\hat{\mu}}_{\alpha}\right| L, l\right\rangle\left\langle l, L\left|\overline{\hat{\mu}_{\gamma}}\right| M, m\right\rangle\left\langle m, M\left|\hat{\mu}_{\delta}\right| 0,0\right\rangle}{\left(\omega_{k K}+i \Gamma_{k K} / 2+\omega_{1}\right)\left(\omega_{l L}-i \Gamma_{l L} / 2-\omega_{2}-\omega_{3}\right)\left(\omega_{m M}-i \Gamma_{m M} / 2-\omega_{3}\right)} \\
& +\frac{\left\langle 0,0\left|\hat{\mu}_{\beta}\right| K, k\right\rangle\left\langle k, K\left|\overline{\hat{\mu}_{\gamma}}\right| L, l\right\rangle\left\langle l, L\left|\overline{\hat{\mu}_{\alpha}}\right| M, m\right\rangle\left\langle m, M\left|\hat{\mu}_{\delta}\right| 0,0\right\rangle}{\left(\omega_{k K}+i \Gamma_{k K} / 2+\omega_{1}\right)\left(\omega_{l L}+i \Gamma_{l L} / 2+\omega_{1}+\omega_{2}\right)\left(\omega_{m M}-i \Gamma_{m M} / 2-\omega_{3}\right)}
\end{aligned}
$$




$$
\begin{aligned}
& \left.+\frac{\left\langle 0,0\left|\hat{\mu}_{\beta}\right| K, k\right\rangle\left\langle k, K\left|\overline{\hat{\mu}_{\gamma}}\right| L, l\right\rangle\left\langle l, L\left|\overline{\hat{\mu}_{\delta}}\right| M, m\right\rangle\left\langle m, M\left|\hat{\mu}_{\alpha}\right| 0,0\right\rangle}{\left(\omega_{k K}+i \Gamma_{k K} / 2+\omega_{1}\right)\left(\omega_{l L}+i \Gamma_{l L} / 2+\omega_{1}+\omega_{2}\right)\left(\omega_{m M}+i \Gamma_{m M} / 2+\omega_{\sigma}\right)}\right) \\
& -\sum_{k, K}{ }^{\prime} \sum_{l, L}{ }^{\prime}\left(\frac{\left\langle 0,0\left|\hat{\mu}_{\alpha}\right| K, k\right\rangle\left\langle k, K\left|\hat{\mu}_{\beta}\right| 0,0\right\rangle\left\langle 0,0\left|\hat{\mu}_{\gamma}\right| L, l\right\rangle\left\langle l, L\left|\hat{\mu}_{\delta}\right| 0,0\right\rangle}{\left(\omega_{k K}-i \Gamma_{k K} / 2-\omega_{\sigma}\right)\left(\omega_{k K}-i \Gamma_{k K} / 2-\omega_{1}\right)\left(\omega_{l L}-i \Gamma_{l L} / 2-\omega_{3}\right)}\right. \\
& +\frac{\left\langle 0,0\left|\hat{\mu}_{\alpha}\right| K, k\right\rangle\left\langle k, K\left|\hat{\mu}_{\beta}\right| 0,0\right\rangle\left\langle 0,0\left|\hat{\mu}_{\gamma}\right| L, l\right\rangle\left\langle l, L\left|\hat{\mu}_{\delta}\right| 0,0\right\rangle}{\left(\omega_{k K}-i \Gamma_{k K} / 2-\omega_{1}\right)\left(\omega_{l L}+i \Gamma_{l L} / 2+\omega_{2}\right)\left(\omega_{l L}-i \Gamma_{l L} / 2-\omega_{3}\right)} \\
& +\frac{\left\langle 0,0\left|\hat{\mu}_{\beta}\right| K, k\right\rangle\left\langle k, K\left|\hat{\mu}_{\alpha}\right| 0,0\right\rangle\left\langle 0,0\left|\hat{\mu}_{\gamma}\right| L, l\right\rangle\left\langle l, L\left|\hat{\mu}_{\delta}\right| 0,0\right\rangle}{\left(\omega_{k K}+i \Gamma_{k K} / 2+\omega_{1}\right)\left(\omega_{k K}+i \Gamma_{k K} / 2+\omega_{\sigma}\right)\left(\omega_{l L}+i \Gamma_{l L} / 2+\omega_{2}\right)} \\
& \left.\left.+\frac{\left\langle 0,0\left|\hat{\mu}_{\beta}\right| K, k\right\rangle\left\langle k, K\left|\hat{\mu}_{\alpha}\right| 0,0\right\rangle\left\langle 0,0\left|\hat{\mu}_{\gamma}\right| L, l\right\rangle\left\langle l, L\left|\hat{\mu}_{\delta}\right| 0,0\right\rangle}{\left(\omega_{k K}+i \Gamma_{k K} / 2+\omega_{1}\right)\left(\omega_{l L}+i \Gamma_{l L} / 2+\omega_{2}\right)\left(\omega_{l L}-i \Gamma_{l L} / 2-\omega_{3}\right)}\right)\right) .
\end{aligned}
$$

In this expression $|K, k\rangle$ is a vibronic wavefunction where $K$ and $k$ refer to electronic and vibrational states, respectively; $\hbar \omega_{k K}$ is the energy of the state $|K, k\rangle$ relative to the ground state $|0,0\rangle$; and the primes now mean the exclusion of the $|0,0\rangle$ state in the summations over all the vibronic states. In distinction to Eq. (1), Eq. (2) does not depend on a fixed nuclear geometry.

In the TPA process, two photons with the same frequency $\omega$ simultaneously induce an excitation from the ground state to some excited state $\Lambda$. The absorption is described by the imaginary part of Eq. (2) with $\omega_{1}=-\omega_{2}=\omega_{3}=\omega$ (and thus $\omega_{\sigma}$ $=\omega$ ). We therefore consider cases where $2 \omega \approx \omega_{l \Lambda}, l$ being the vibrational state index of interest. For this resonance (or near-resonance) all the terms in Eq. (2) can be considered insignificant in comparison with those having the denominator $\left(\omega_{l \Lambda}-i \Gamma_{l \Lambda} / 2-2 \omega\right)$. Thus, only the first two terms in Eq. (2) are important and all the terms with $L \neq \Lambda$ can be discarded. When $\omega$ is not close to any of the intermediate-state frequencies $\omega_{k K}\left(\omega_{m M}\right)$ then $\Gamma_{k K}\left(\Gamma_{m M}\right)$ can be neglected. We can also replace the remaining damping factors $\Gamma_{l \Lambda}$ simply by $\Gamma$ by ignoring their vibrational dependence. This leads to the formula

$\operatorname{Im} \gamma_{\alpha \beta \gamma \delta}(-\omega ; \omega,-\omega, \omega)=\frac{\Gamma \hbar^{-3}}{2} \sum P_{\alpha \gamma} \sum P_{\beta \delta} \sum_{k, K}{ }^{\prime} \sum_{m, M}{ }^{\prime} \sum_{l} \frac{\left\langle 0,0\left|\hat{\mu}_{\alpha}\right| K, k\right\rangle\left\langle k, K\left|\overline{\hat{\mu}}_{\gamma}\right| \Lambda, l\right\rangle\left\langle l, \Lambda\left|\overline{\hat{\mu}_{\beta}}\right| M, m\right\rangle\left\langle m, M\left|\hat{\mu}_{\delta}\right| 0,0\right\rangle}{\left(\left(\omega_{l \Lambda}-2 \omega\right)^{2}+\Gamma^{2} / 4\right)\left(\omega_{k K}-\omega\right)\left(\omega_{m M}-\omega\right)}$

where $\Sigma P_{\alpha \gamma}$, for example, means a sum over the two terms obtained by permuting $\hat{\mu}_{\alpha}$ and $\hat{\mu}_{\gamma}$; note that the sum over the vibrational levels of the $\Lambda$ excited state (index $l$ ) includes the ground vibrational state $(l=0)$.

Following the procedure adopted in BK, we now assume that for any intermediate state not involving the electronic ground state, the vibrational energy component can be ignored and we can replace $\omega_{k K}$ by $\omega_{0 K}$, etc., provided $\omega$ is not close to any $\omega_{k K}$. This leads to

$$
\begin{aligned}
\operatorname{Im} \gamma_{\alpha \beta \gamma \delta}(-\omega ; \omega,-\omega, \omega)= & \frac{\hbar}{2} \sum P_{\alpha \gamma} \sum P_{\beta \delta} \sum_{l} \hbar^{-2}\left[\left(\omega_{l \Lambda}-2 \omega\right)^{2}+\Gamma^{2} / 4\right]^{-1} \\
& \times\left\{\sum_{K}{ }^{\prime} \sum_{M}{ }^{\prime} \frac{\left\langle 0^{0}\left|\left\langle 0\left|\hat{\mu}_{\alpha}\right| K\right\rangle\left\langle K\left|\overline{\hat{\mu}_{\gamma}}\right| \Lambda\right\rangle\right| l^{\Lambda}\right\rangle\left\langle l^{\Lambda}\left|\left\langle\Lambda\left|\overline{\mu_{\beta}}\right| M\right\rangle\left\langle M\left|\hat{\mu}_{\delta}\right| 0\right\rangle\right| 0^{0}\right\rangle}{\hbar^{2}\left(\omega_{0 K}-\omega\right)\left(\omega_{0 M}-\omega\right)}\right. \\
& +\sum_{k}{ }^{\prime} \sum_{M}{ }^{\prime} \frac{\left\langle 0^{0}\left|\left\langle 0\left|\hat{\mu}_{\alpha}\right| 0\right\rangle\right| k^{0}\right\rangle\left\langle k^{0}\left|\left\langle 0\left|\hat{\mu}_{\gamma}\right| \Lambda\right\rangle\right| l^{\Lambda}\right\rangle\left\langle l^{\Lambda}\left|\left\langle\Lambda\left|\overline{\mu_{\beta}}\right| M\right\rangle\left\langle M\left|\hat{\mu}_{\delta}\right| 0\right\rangle\right| 0^{0}\right\rangle}{\hbar^{2}\left(\omega_{k 0}-\omega\right)\left(\omega_{0 M}-\omega\right)} \\
& +\sum_{K}{ }^{\prime} \sum_{m}{ }^{\prime} \frac{\left\langle 0^{0}\left|\left\langle 0\left|\hat{\mu}_{\alpha}\right| K\right\rangle\left\langle K\left|\overline{\hat{\mu}_{\gamma}}\right| \Lambda\right\rangle\right| l^{\Lambda}\right\rangle\left\langle l^{\Lambda}\left|\left\langle\Lambda\left|\hat{\mu}_{\beta}\right| 0\right\rangle\right| m^{0}\right\rangle\left\langle m^{0}\left|\left\langle 0\left|\hat{\mu}_{\delta}\right| 0\right\rangle\right| 0^{0}\right\rangle}{\hbar^{2}\left(\omega_{0 K}-\omega\right)\left(\omega_{m 0}-\omega\right)} \\
& +\sum_{k}{ }^{\prime} \sum_{m}{ }^{\prime} \frac{\left\langle 0^{0}\left|\left\langle 0\left|\hat{\mu}_{\alpha}\right| 0\right\rangle\right| k^{0}\right\rangle\left\langle k^{0}\left|\left\langle 0\left|\hat{\mu}_{\gamma}\right| \Lambda\right\rangle\right| l^{\Lambda}\right\rangle\left\langle l^{\Lambda}\left|\left\langle\Lambda\left|\hat{\mu}_{\beta}\right| 0\right\rangle\right| m^{0}\right\rangle\left\langle m^{0}\left|\left\langle 0\left|\hat{\mu}_{\delta}\right| 0\right\rangle\right| 0^{0}\right\rangle}{\hbar^{2}\left(\omega_{k 0}-\omega\right)\left(\omega_{m 0}-\omega\right)},
\end{aligned}
$$


where $\left|0^{0}\right\rangle$ and $\left|l^{\Lambda}\right\rangle$ represent the ground vibrational wavefunction of the ground electronic state and the $l$ th vibrational wavefunction of the $\Lambda$ electronic state (the one to which the two-photon absorption is taking place).

Equation (4) can be simplified by defining electronic transition dipole and polarizability functions as

$$
\mu_{\alpha}^{0 K}(\mathbf{R})=\left\langle 0\left|\hat{\mu}_{\alpha}\right| K\right\rangle
$$

and

$$
\begin{aligned}
\alpha_{\alpha \gamma}^{0 \Lambda}(\mathbf{R}, \omega) & =\sum P_{\alpha \gamma} \hbar^{-1} \sum_{K}{ }^{\prime} \frac{\left\langle 0\left|\overline{\hat{\mu}_{\alpha}}\right| K\right\rangle\left\langle K\left|\overline{\hat{\mu}_{\gamma}}\right| \Lambda\right\rangle}{\omega_{0 K}-\omega} \\
& =\sum P_{\alpha \gamma} \hbar^{-1} \sum_{K}{ }^{\prime} \frac{\left\langle 0\left|\hat{\mu}_{\alpha}\right| K\right\rangle\left\langle K\left|\overline{\hat{\mu}_{\gamma}}\right| \Lambda\right\rangle}{\omega_{0 K}-\omega} \\
\alpha_{\beta \delta}^{\Lambda 0}(\mathbf{R}, \omega) & =\sum P_{\beta \delta} \hbar^{-1} \sum_{K}{ }^{\prime} \frac{\left\langle\Lambda\left|\overline{\hat{\mu}_{\beta}}\right| K\right\rangle\left\langle K\left|\overline{\hat{\mu}_{\delta}}\right| 0\right\rangle}{\omega_{0 K}-\omega} \\
& =\sum P_{\beta \delta} \hbar^{-1} \sum_{K}{ }^{\prime} \frac{\left\langle\Lambda\left|\overline{\hat{\mu}_{\beta}}\right| K\right\rangle\left\langle K\left|\hat{\mu}_{\delta}\right| 0\right\rangle}{\omega_{0 K}-\omega}
\end{aligned}
$$

where $\mathbf{R}$ indicates the molecular geometry. Again, following past procedures, in this case Refs. 33 and 34, we replace in Eqs. (6) and (7) $\hbar \omega_{0 K}$ by $\hbar \omega_{K}$, the latter being the energy of the $K$ th electronic state as a function of $\mathbf{R}$. This leads to the following clamped nucleus electronic transition polarizability functions:

$$
\begin{aligned}
\alpha_{\alpha \gamma}^{0 \Lambda}(\mathbf{R}, \omega) & =\sum P_{\alpha \gamma} \hbar^{-1} \sum_{K}{ }^{\prime} \frac{\left\langle 0\left|\hat{\hat{\mu}}_{\alpha}\right| K\right\rangle\left\langle K\left|\hat{\hat{\mu}}_{\gamma}\right| \Lambda\right\rangle}{\omega_{0 K}-\omega} \\
& \cong \sum P_{\alpha \gamma} \hbar^{-1} \sum_{K}{ }^{\prime} \frac{\left\langle 0\left|\hat{\hat{\mu}}_{\alpha}\right| K\right\rangle\left\langle K\left|\hat{\hat{\mu}}_{\gamma}\right| \Lambda\right\rangle}{\omega_{K}-\omega}, \\
\alpha_{\beta \delta}^{\Lambda 0}(\mathbf{R}, \omega) & =\sum P_{\beta \delta} \hbar^{-1} \sum_{K}{ }^{\prime} \frac{\left\langle\Lambda\left|\hat{\mu}_{\beta}\right| K\right\rangle\left\langle K\left|\hat{\mu}_{\delta}\right| 0\right\rangle}{\omega_{0 K}-\omega} \\
& \cong \sum P_{\beta \delta} \hbar^{-1} \sum_{K}{ }^{\prime} \frac{\left\langle\Lambda\left|\hat{\mu}_{\beta}\right| K\right\rangle\left\langle K \mid \hat{\hat{\mu}}_{\delta} 0\right\rangle}{\omega_{K}-\omega} .
\end{aligned}
$$

Combining Eqs. (4), (5), (8) and (9) we get

$$
\begin{aligned}
\operatorname{Im} \gamma_{\alpha \beta \gamma \delta}(-\omega ; \omega,-\omega, \omega)= & \frac{\hbar \Gamma}{2} \sum_{l} \hbar^{-2}\left[\left(\omega_{l \Lambda}-2 \omega\right)^{2}+\Gamma^{2} / 4\right]^{-1}\left\{\left\langle 0^{0}\left|\alpha_{\alpha \gamma}^{0 \Lambda}(\mathbf{R}, \omega)\right| l^{\Lambda}\right\rangle\left\langle l^{\Lambda}\left|\alpha_{\beta \delta}^{\Lambda 0}(\mathbf{R}, \omega)\right| 0^{0}\right\rangle\right. \\
& +\sum P_{\alpha \gamma} \sum_{k}{ }^{\prime} \frac{\left\langle 0^{0}\left|\mu_{\alpha}^{00}(\mathbf{R})\right| k^{0}\right\rangle\left\langle k^{0}\left|\mu_{\gamma}^{0 \Lambda}(\mathbf{R})\right| l^{\Lambda}\right\rangle\left\langle l^{\Lambda}\left|\alpha_{\beta \delta}^{\Lambda 0}(\mathbf{R}, \omega)\right| 0^{0}\right\rangle}{\hbar\left(\omega_{k 0}-\omega\right)} \\
& +\sum P_{\beta \delta} \sum_{m}{ }^{\prime} \frac{\left\langle 0^{0}\left|\alpha_{\alpha \gamma}^{0 \Lambda}(\mathbf{R}, \omega)\right| l^{\Lambda}\right\rangle\left\langle l^{\Lambda}\left|\mu_{\beta}^{\Lambda 0}(\mathbf{R})\right| m^{0}\right\rangle\left\langle m^{0}\left|\mu_{\delta}^{00}(\mathbf{R})\right| 0^{0}\right\rangle}{\hbar\left(\omega_{m 0}-\omega\right)} \\
& \left.+\sum P_{\alpha \gamma} \sum P_{\beta \delta} \sum_{k}{ }^{\prime} \sum_{m}{ }^{\prime} \frac{\left\langle 0^{0}\left|\mu_{\alpha}^{00}(\mathbf{R})\right| k^{0}\right\rangle\left\langle k^{0}\left|\mu_{\gamma}^{0 \Lambda}(\mathbf{R})\right| l^{\Lambda}\right\rangle\left\langle l^{\Lambda}\left|\mu_{\beta}^{\Lambda 0}(\mathbf{R})\right| m^{0}\right\rangle\left\langle m^{0}\left|\mu_{\delta}^{00}(\mathbf{R})\right| 0^{0}\right\rangle}{\hbar^{2}\left(\omega_{k 0}-\omega\right)\left(\omega_{m 0}-\omega\right)}\right\}
\end{aligned}
$$

Note that the index $l$ extends over all the vibrational states of electronic state $\Lambda$. Equation (10) is our working formula.

A compact form of this expression, à la the square bracket notation of $\mathrm{BK}$, is

$$
\operatorname{Im} \gamma_{\alpha \beta \gamma \delta}(-\omega ; \omega,-\omega, \omega)=\left\{\alpha^{2}\right\}+\left\{\mu^{2} \alpha\right\}+\left\{\mu^{4}\right\},
$$

where $\left\{\alpha^{2}\right\},\left\{\mu^{2} \alpha\right\}$ and $\left\{\mu^{4}\right\}$ correspond to the first, second and third (combined) and fourth terms of Eq. (10), respectively. We call $\left\{\mu^{2} \alpha\right\}$ and $\left\{\mu^{4}\right\}$ pure vibrational terms because, unlike the $\left\{\alpha^{2}\right\}$ term, they would not be present except for vibrational motion on the potential energy surface of the ground electronic state. The $\mu^{2}$ factor in $\left\{\mu^{2} \alpha\right\}$ is similar to the vibrational polarizability at frequency $\omega$ that occurs in the nonresonant case, but here we are dealing with a transition polarizability between the ground and two-photon elec- tronic states; the $\left\{\mu^{4}\right\}$ term is simply a product of two such vibrational transition polarizabilities. In the clamped nucleus approximation the $\left\{\alpha^{2}\right\}$ term would give rise to a pure electronic TPA. However, Eq. (10) explicitly takes into account the vibrational wavefunctions and energies associated with the two-photon electronic state.

As a matter of interest, and for purposes of comparison, we can also carry out the previous methodology starting from the electronic formula in Eq. (1). With the same assumptions as before, we then arrive at

$\operatorname{Im} \gamma_{\alpha \beta \gamma \delta}(-\omega ; \omega,-\omega, \omega)=\frac{\hbar^{-1} \Gamma}{2} \frac{\alpha_{\alpha \gamma}^{0 \Lambda}(\mathbf{R}, \omega) \alpha_{\beta \delta}^{\Lambda 0}(\mathbf{R}, \omega)}{\left(\omega_{\Lambda}-2 \omega\right)^{2}+\Gamma^{2} / 4}$ 
We consider this to be the pure electronic TPA with all vibrational effects ignored. Normally Eq. (12) would be evaluated at $\mathbf{R}=\mathbf{R}_{0}$, the equilibrium nuclear geometry of the ground electronic state and with $\hbar \omega_{\Lambda}$ as a function of $\mathbf{R}$. With certain assumptions, see Ref. 34, Eq. (12) can also be derived from Eq. (2). Equation (12) can be compared with the combination of Eqs. (2) and (4) in Ref. 26 by taking $\omega$ $=\omega_{\Lambda} / 2$ in their expression for the transition polarizability function and recognizing that the summations in Eq. (4) of Ref. 26 include the ground state-this is in place of using fluctuation dipole moment operators (see Ref. 35 for a more complete explanation). Also their damping factor is different from that used here.

In order to relate to experimental data (gas or liquid phase) we must carry out orientational averaging by using the formula ${ }^{26}$

$$
\begin{aligned}
\gamma^{\mathrm{TP}}= & 2 \sum_{\alpha, \beta}\left[\operatorname{Im} \gamma_{\alpha \alpha \beta \beta}(-\omega ; \omega,-\omega, \omega)\right. \\
& +\operatorname{Im} \gamma_{\alpha \beta \alpha \beta}(-\omega ; \omega,-\omega, \omega) \\
& \left.+\operatorname{Im} \gamma_{\alpha \beta \beta \alpha}(-\omega ; \omega,-\omega, \omega)\right]
\end{aligned}
$$

The relation between $\gamma^{\mathrm{TP}}$ and the two photon cross-section per photon $\sigma^{\mathrm{TP}}$ is

$$
\sigma^{\mathrm{TP}}=\frac{4 \pi^{3}}{15} \frac{\alpha^{2} \hbar^{3} \omega^{2}}{e^{4}} \gamma^{\mathrm{TP}}
$$

where $\alpha$ is the fine-structure constant. We first evaluate $\sigma^{\mathrm{TP}}$ in atomic units and then multiply by $(0.529177$ $\times 10^{-8} \mathrm{~cm} /$ a.u. $)^{4}\left(2.418884 \times 10^{-17}\right.$ s/a.u. $)$ to obtain $\sigma^{\mathrm{TP}}$ in the conventional units of $\mathrm{cm}^{4} \mathrm{~s} /$ photon.

\section{DIATOMICS-PERTURBATION THEORY}

We now face the problem of evaluating the integrals which occur in Eq. (10). We will, at this stage, restrict ourselves to diatomic molecules though much, but not all, of the formalism can be carried over to polyatomics. Of course for diatomic molecules vibrational wavefunctions can be found directly with Le Roy's program ${ }^{29}$ and the needed integrals evaluated numerically. In fact we will do this in order to benchmark our more approximate treatment which now follows and which is the only one feasible for polyatomic molecules.

This approximate treatment is based on perturbation theory. First we make a Taylor series expansion of the ground state dipole moment function as well as the transition dipole and polarizability functions:

$$
\begin{aligned}
\mu_{\alpha}^{00}(\mathbf{R})= & \mu_{\alpha}^{00}\left(\mathbf{R}_{\mathbf{0}}\right)+\left(\frac{\partial \mu_{\alpha}^{00}(\mathbf{R})}{\partial Q^{0}}\right)_{\mathbf{R}_{\mathbf{0}}} Q^{0} \\
& +\frac{1}{2 !}\left(\frac{\partial^{2} \mu_{\alpha}^{00}(\mathbf{R})}{\partial\left(Q^{0}\right)^{2}}\right)_{\mathbf{R}_{\mathbf{0}}}\left(Q^{0}\right)^{2} \\
& +\frac{1}{3 !}\left(\frac{\partial^{3} \mu_{\alpha}^{00}(\mathbf{R})}{\partial\left(Q^{0}\right)^{3}}\right)_{\mathbf{R}_{\mathbf{0}}}\left(Q^{0}\right)^{3}+\cdots, \\
\mu_{\alpha}^{0 \Lambda}(\mathbf{R})= & \mu_{\alpha}^{0 \Lambda}\left(\mathbf{R}_{\mathbf{0}}\right)+\left(\frac{\partial \mu_{\alpha}^{0 \Lambda}(\mathbf{R})}{\partial Q^{0}}\right)_{\mathbf{R}_{\mathbf{0}}} Q^{0} \\
& +\frac{1}{2 !}\left(\frac{\partial^{2} \mu_{\alpha}^{0 \Lambda}(\mathbf{R})}{\partial\left(Q^{0}\right)^{2}}\right)_{\mathbf{R}_{\mathbf{0}}}\left(Q^{0}\right)^{2} \\
& +\frac{1}{3 !}\left(\frac{\partial^{3} \mu_{\alpha}^{0 \Lambda}(\mathbf{R})}{\partial\left(Q^{0}\right)^{3}}\right)_{\mathbf{R}_{\mathbf{0}}}\left(Q^{0}\right)^{3}+\cdots
\end{aligned}
$$

$$
\begin{aligned}
\alpha_{\alpha \gamma}^{0 \Lambda}(\mathbf{R}, \omega)= & \alpha_{\alpha \gamma}^{0 \Lambda}\left(\mathbf{R}_{\mathbf{0}}\right)+\left(\frac{\partial \alpha_{\alpha \gamma}^{0 \Lambda}(\mathbf{R}, \omega)}{\partial Q^{0}}\right)_{\mathbf{R}_{\mathbf{0}}} Q^{0} \\
& +\frac{1}{2 !}\left(\frac{\partial^{2} \alpha_{\alpha \gamma}^{0 \Lambda}(\mathbf{R}, \omega)}{\partial\left(Q^{0}\right)^{2}}\right)_{\mathbf{R}_{\mathbf{0}}}\left(Q^{0}\right)^{2} \\
& +\frac{1}{3 !}\left(\frac{\partial^{3} \alpha_{\alpha \gamma}^{0 \Lambda}(\mathbf{R}, \omega)}{\partial\left(Q^{0}\right)^{3}}\right)_{\mathbf{R}_{\mathbf{0}}}\left(Q^{0}\right)^{3}+\cdots,
\end{aligned}
$$

where $Q^{0}$ is the electronic ground state normal coordinate and $\mathbf{R}_{0}$ identifies the equilibrium nuclear geometry of that state. For a diatomic molecule the normal coordinate of an excited electronic state differs from $Q^{0}$ only by a constant. The third- and higher-order terms in these series constitute electrical anharmonicity. With these expansions, the integrals required in Eq. (10) can be written as

$$
\begin{aligned}
\left\langle 0^{0}\left|\mu_{\alpha}^{00}(\mathbf{R})\right| k^{0}\right\rangle= & \left(\frac{\partial \mu_{\alpha}^{00}(\mathbf{R})}{\partial Q^{0}}\right)_{\mathbf{R}_{\mathbf{0}}}\left\langle 0^{0}\left|Q^{0}\right| k^{0}\right\rangle \\
& +\frac{1}{2 !}\left(\frac{\partial^{2} \mu_{\alpha}^{00}(\mathbf{R})}{\partial\left(Q^{0}\right)^{2}}\right)_{\mathbf{R}_{\mathbf{0}}}\left\langle 0^{0}\left|\left(Q^{0}\right)^{2}\right| k^{0}\right\rangle \\
& +\frac{1}{3 !}\left(\frac{\partial^{3} \mu_{\alpha}^{00}(\mathbf{R})}{\partial\left(Q^{0}\right)^{3}}\right)_{\mathbf{R}_{\mathbf{0}}}\left\langle 0^{0}\left|\left(Q^{0}\right)^{3}\right| k^{0}\right\rangle+\cdots,
\end{aligned}
$$




$$
\begin{aligned}
\left\langle k^{0}\left|\mu_{\alpha}^{0 \Lambda}(\mathbf{R})\right| l^{\Lambda}\right\rangle= & \mu_{\alpha}^{0 \Lambda}\left(\mathbf{R}_{\mathbf{0}}\right)\left\langle k^{0} \mid l^{\Lambda}\right\rangle \\
& +\left(\frac{\partial \mu_{\alpha}^{0 \Lambda}(\mathbf{R})}{\partial Q^{0}}\right)_{\mathbf{R}_{\mathbf{0}}}\left\langle k^{0}\left|Q^{0}\right| l^{\Lambda}\right\rangle \\
& +\frac{1}{2 !}\left(\frac{\partial^{2} \mu_{\alpha}^{0 \Lambda}(\mathbf{R})}{\partial\left(Q^{0}\right)^{2}}\right)_{\mathbf{R}_{\mathbf{0}}}\left\langle k^{0}\left|\left(Q^{0}\right)^{2}\right| l^{\Lambda}\right\rangle \\
& +\frac{1}{3 !}\left(\frac{\partial^{3} \mu_{\alpha}^{0 \Lambda}(\mathbf{R})}{\partial\left(Q^{0}\right)^{3}}\right)_{\mathbf{R}_{\mathbf{0}}}\left\langle k^{0}\left|\left(Q^{0}\right)^{3}\right| l^{\Lambda}\right\rangle+\cdots,
\end{aligned}
$$

$$
\begin{aligned}
\left\langle 0^{0}\left|\alpha_{\alpha \gamma}^{0 \Lambda}(\mathbf{R}, \omega)\right| l^{\Lambda}\right\rangle= & \alpha_{\alpha \gamma}^{0 \Lambda}\left(\mathbf{R}_{\mathbf{0}}, \omega\right)\left\langle 0^{0} \mid l^{\Lambda}\right\rangle \\
& +\left(\frac{\partial \alpha_{\alpha \gamma}^{0 \Lambda}(\mathbf{R}, \omega)}{\partial Q^{0}}\right)_{\mathbf{R}_{\mathbf{0}}}\left\langle 0^{0}\left|Q^{0}\right| l^{\Lambda}\right\rangle \\
& +\frac{1}{2 !}\left(\frac{\partial^{2} \alpha_{\alpha \gamma}^{0 \Lambda}(\mathbf{R}, \omega)}{\partial\left(Q^{0}\right)^{2}}\right)_{\mathbf{R}_{\mathbf{0}}}\left\langle 0^{0}\left|\left(Q^{0}\right)^{2}\right| l^{\Lambda}\right\rangle \\
& +\frac{1}{3 !}\left(\frac{\partial^{3} \alpha_{\alpha \gamma}^{0 \Lambda}(\mathbf{R}, \omega)}{\partial\left(Q^{0}\right)^{3}}\right)_{\mathbf{R}_{\mathbf{0}}}\left\langle 0^{0}\left|\left(Q^{0}\right)^{3}\right| l^{\Lambda}\right\rangle \\
& +\cdots
\end{aligned}
$$

In Eq. (18) use has been made of the fact that $\left\langle 0^{0} \mid k^{0}\right\rangle=0$ for $k$ different from zero.

Not too far from the appropriate equilibrium geometry the potential energy functions for the electronic ground and $\Lambda$ excited state can also be expanded as

$$
\begin{aligned}
V^{0}(\mathbf{R})= & V^{0}\left(\mathbf{R}_{\mathbf{0}}\right)+\frac{1}{2 !}\left(\frac{\partial^{2} V^{0}(\mathbf{R})}{\partial\left(Q^{0}\right)^{2}}\right)_{\mathbf{R}_{\mathbf{0}}}\left(Q^{0}\right)^{2} \\
& +\frac{1}{3 !}\left(\frac{\partial^{3} V^{0}(\mathbf{R})}{\partial\left(Q^{0}\right)^{3}}\right)_{\mathbf{R}_{\mathbf{0}}}\left(Q^{0}\right)^{3} \\
& +\frac{1}{4 !}\left(\frac{\partial^{4} V^{0}(\mathbf{R})}{\partial\left(Q^{0}\right)^{4}}\right)_{\mathbf{R}_{\mathbf{0}}}\left(Q^{0}\right)^{4}+\cdots, \\
V^{\Lambda}(\mathbf{R})= & V^{\Lambda}\left(\mathbf{R}_{\Lambda}\right)+\frac{1}{2 !}\left(\frac{\partial^{2} V^{\Lambda}(\mathbf{R})}{\partial\left(Q^{\Lambda}\right)^{2}}\right)_{\mathbf{R}_{\Lambda}}\left(Q^{\Lambda}\right)^{2} \\
& +\frac{1}{3 !}\left(\frac{\partial^{3} V^{\Lambda}(\mathbf{R})}{\partial\left(Q^{\Lambda}\right)^{3}}\right)_{\mathbf{R}_{\Lambda}}\left(Q^{\Lambda}\right)^{3} \\
& +\frac{1}{4 !}\left(\frac{\partial^{4} V^{\Lambda}(\mathbf{R})}{\partial\left(Q^{\Lambda}\right)^{4}}\right)_{\mathbf{R}_{\Lambda}}\left(Q^{\Lambda}\right)^{4}+\cdots,
\end{aligned}
$$

where $Q^{\Lambda}$ is the normal coordinate of the $\Lambda$-excited state. The third and higher terms introduce mechanical anharmonicity. With these expansions, the vibrational wavefunctions can be expressed in terms of harmonic oscillator functions $\left|k_{H}^{0}\right\rangle$ and $\left|l_{H}^{\Lambda}\right\rangle$ as $^{36}$

$$
\begin{aligned}
\left|k^{0}\right\rangle=\mid & \left.k_{H}^{0}\right\rangle-\sum_{j \neq k} \frac{\left\langle k_{H}^{0}\left|V^{0(1)}\right| j_{H}^{0}\right\rangle\left|j_{H}^{0}\right\rangle}{\hbar\left(\omega_{j 0}^{H}-\omega_{k 0}^{H}\right)} \\
& +\sum_{i, j \neq k} \frac{\left\langle k_{H}^{0}\left|V^{0(1)}\right| j_{H}^{0}\right\rangle\left\langle j_{H}^{0}\left|V^{0(1)}\right| i_{H}^{0}\right\rangle\left|i_{H}^{0}\right\rangle}{\hbar^{2}\left(\omega_{j 0}^{H}-\omega_{k 0}^{H}\right)\left(\omega_{i 0}^{H}-\omega_{k 0}^{H}\right)} \\
& -\sum_{j \neq k} \frac{\left\langle k_{H}^{0}\left|V^{0(2)}\right| j_{H}^{0}\right\rangle\left|j_{H}^{0}\right\rangle}{\hbar\left(\omega_{j 0}^{H}-\omega_{k 0}^{H}\right)} \\
& -\frac{1}{2} \sum_{j \neq k} \frac{\left\langle k_{H}^{0}\left|V^{0(1)}\right| j_{H}^{0}\right\rangle\left\langle j_{H}^{0}\left|V^{0(1)}\right| k_{H}^{0}\right\rangle\left|k_{H}^{0}\right\rangle}{\hbar^{2}\left(\omega_{j 0}^{H}-\omega_{k 0}^{H}\right)^{2}}+\cdots, \\
\left|l^{\Lambda}\right\rangle=\mid & \left|l_{H}^{\Lambda}\right\rangle-\sum_{m \neq l} \frac{\left\langle l_{H}^{\Lambda}\left|V^{\Lambda(1)}\right| m_{H}^{\Lambda}\right\rangle\left|m_{H}^{\Lambda}\right\rangle}{\hbar\left(\omega_{m \Lambda}^{H}-\omega_{l \Lambda}^{H}\right)} \\
& +\sum_{m, n \neq l} \frac{\left\langle l_{H}^{\Lambda}\left|V^{\Lambda(1)}\right| m_{H}^{\Lambda}\right\rangle\left\langle m_{H}^{\Lambda}\left|V^{\Lambda(1)}\right| n_{H}^{\Lambda}\right\rangle\left|n_{H}^{\Lambda}\right\rangle}{\hbar^{2}\left(\omega_{m \Lambda}^{H}-\omega_{l \Lambda}^{H}\right)\left(\omega_{n \Lambda}^{H}-\omega_{l \Lambda}^{H}\right)} \\
& -\sum_{m \neq l} \frac{\left\langle l_{H}^{\Lambda}\left|V^{\Lambda(2)}\right| m_{H}^{\Lambda}\right\rangle\left|m_{H}^{\Lambda}\right\rangle}{\hbar\left(\omega_{m \Lambda}^{H}-\omega_{l \Lambda}^{H}\right)} \\
& -\frac{1}{2} \sum_{m \neq l} \frac{\left\langle l_{H}^{\Lambda}\left|V^{\Lambda(1)}\right| m_{H}^{\Lambda}\right\rangle\left\langle m_{H}^{\Lambda}\left|V^{\Lambda(1)}\right| l_{H}^{\Lambda}\right\rangle\left|l_{H}^{\Lambda}\right\rangle}{\hbar^{2}\left(\omega_{m \Lambda}^{H}-\omega_{l \Lambda}^{H}\right)^{2}}+\cdots, \\
&
\end{aligned}
$$

where

$$
\begin{aligned}
& V^{0(1)}=\frac{1}{3 !}\left(\frac{\partial^{3} V^{0}(\mathbf{R})}{\partial\left(Q^{0}\right)^{3}}\right)_{\mathbf{R}_{0}}\left(Q^{0}\right)^{3} \\
& V^{0(2)}=\frac{1}{4 !}\left(\frac{\partial^{4} V^{0}(\mathbf{R})}{\partial\left(Q^{0}\right)^{4}}\right)_{\mathbf{R}_{0}}\left(Q^{0}\right)^{4}, \\
& V^{\Lambda(1)}=\frac{1}{3 !}\left(\frac{\partial^{3} V^{\Lambda}(\mathbf{R})}{\partial\left(Q^{\Lambda}\right)^{3}}\right)_{\mathbf{R}_{\Lambda}}\left(Q^{\Lambda}\right)^{3}, \\
& V^{\Lambda(2)}=\frac{1}{4 !}\left(\frac{\partial^{4} V^{\Lambda}(\mathbf{R})}{\partial\left(Q^{\Lambda}\right)^{4}}\right)_{\mathbf{R}_{\Lambda}}\left(Q^{\Lambda}\right)^{4},
\end{aligned}
$$

and $\hbar \omega_{j 0}^{H}$ is the energy of the state $\left|j^{0}\right\rangle$ relative to the ground state calculated within the harmonic oscillator approximation.

The individual integrals on the right of Eq. (19), for example, can be written quite generally as 


$$
\begin{aligned}
& \left\langle k^{0}\left|\left(Q^{0}\right)^{t}\right| l^{\Lambda}\right\rangle=\left\langle k_{H}^{0}\left|\left(Q^{0}\right)^{t}\right| l_{H}^{\Lambda}\right\rangle-\frac{1}{3 !}\left(\frac{\partial^{3} V^{0}(\mathbf{R})}{\partial\left(Q^{0}\right)^{3}}\right)_{\mathbf{R}_{\mathbf{0}}} \sum_{j \neq k}\left[\frac{\left\langle j_{H}^{0}\left|\left(Q^{0}\right)^{3}\right| k_{H}^{0}\right\rangle\left\langle j_{H}^{0}\left|\left(Q^{0}\right)^{t}\right| l_{H}^{\Lambda}\right\rangle}{\hbar\left(\omega_{j 0}^{H}-\omega_{k 0}^{H}\right)}\right]-\frac{1}{3 !}\left(\frac{\partial^{3} V^{\Lambda}(\mathbf{R})}{\partial\left(Q^{\Lambda}\right)^{3}}\right)_{\mathbf{R}_{\Lambda}} \\
& \times \sum_{m \neq l}\left[\frac{\left\langle l_{H}^{\Lambda}\left|\left(Q^{\Lambda}\right)^{3}\right| m_{H}^{\Lambda}\right\rangle\left\langle k_{H}^{0}\left|\left(Q^{0}\right)^{t}\right| m_{H}^{\Lambda}\right\rangle}{\hbar\left(\omega_{m \Lambda}^{H}-\omega_{l \Lambda}^{H}\right)}\right]-\frac{1}{4 !}\left(\frac{\partial^{4} V^{0}(\mathbf{R})}{\partial\left(Q^{0}\right)^{4}}\right)_{\mathbf{R}_{\mathbf{0}}} \\
& \times \sum_{j \neq k}\left[\frac{\left\langle j_{H}^{0}\left|\left(Q^{0}\right)^{4}\right| k_{H}^{0}\right\rangle\left\langle j_{H}^{0}\left|\left(Q^{0}\right)^{t}\right| l_{H}^{\Lambda}\right\rangle}{\hbar\left(\omega_{j 0}^{H}-\omega_{k 0}^{H}\right)}\right]-\frac{1}{4 !}\left(\frac{\partial^{4} V^{\Lambda}(\mathbf{R})}{\partial\left(Q^{\Lambda}\right)^{4}}\right)_{\mathbf{R}_{\Lambda}} \\
& \times \sum_{m \neq l}\left[\frac{\left\langle l_{H}^{\Lambda}\left|\left(Q^{\Lambda}\right)^{4}\right| m_{H}^{\Lambda}\right\rangle\left\langle k_{H}^{0}\left|\left(Q^{0}\right)^{t}\right| m_{H}^{\Lambda}\right\rangle}{\hbar\left(\omega_{m \Lambda}^{H}-\omega_{l \Lambda}^{H}\right)}\right]+\frac{1}{(3 !)^{2}}\left(\frac{\partial^{3} V^{0}(\mathbf{R})}{\partial\left(Q^{0}\right)^{3}}\right)_{\mathbf{R}_{\mathbf{0}}}\left(\frac{\partial^{3} V^{\Lambda}(\mathbf{R})}{\partial\left(Q^{\Lambda}\right)^{3}}\right)_{\mathbf{R}_{\Lambda}} \\
& \times \sum_{j \neq k, m \neq l}\left[\frac{\left\langle j_{H}^{0}\left|\left(Q^{0}\right)^{3}\right| k_{H}^{0}\right\rangle\left\langle l_{H}^{\Lambda}\left|\left(Q^{\Lambda}\right)^{3}\right| m_{H}^{\Lambda}\right\rangle\left\langle j_{H}^{0}\left|\left(Q^{0}\right)^{t}\right| m_{H}^{\Lambda}\right\rangle}{\hbar^{2}\left(\omega_{j 0}^{H}-\omega_{k 0}^{H}\right)\left(\omega_{m \Lambda}^{H}-\omega_{l \Lambda}^{H}\right)}\right]+\frac{1}{(3 !)^{2}}\left(\frac{\partial^{3} V^{0}(\mathbf{R})}{\partial\left(Q^{0}\right)^{3}}\right)_{\mathbf{R}_{\mathbf{0}}}^{2} \\
& \times \sum_{i, j \neq k}\left[\frac{\left\langle i_{H}^{0}\left|\left(Q^{0}\right)^{3}\right| j_{H}^{0}\right\rangle\left\langle j_{H}^{0}\left|\left(Q^{0}\right)^{3}\right| k_{H}^{0}\right\rangle\left\langle i_{H}^{0}\left|\left(Q^{0}\right)^{t}\right| l_{H}^{\Lambda}\right\rangle}{\hbar^{2}\left(\omega_{i 0}^{H}-\omega_{k 0}^{H}\right)\left(\omega_{j 0}^{H}-\omega_{k 0}^{H}\right)}\right]-\frac{1}{2(3 !)^{2}}\left(\frac{\partial^{3} V^{0}(\mathbf{R})}{\partial\left(Q^{0}\right)^{3}}\right)_{\mathbf{R}_{\mathbf{0}}}^{2} \\
& \times \sum_{j \neq k}\left[\frac{\left\langle k_{H}^{0}\left|\left(Q^{0}\right)^{3}\right| j_{H}^{0}\right\rangle\left\langle j_{H}^{0}\left|\left(Q^{0}\right)^{3}\right| k_{H}^{0}\right\rangle}{\hbar^{2}\left(\omega_{j 0}^{H}-\omega_{k 0}^{H}\right)^{2}}\right]\left\langle k_{H}^{0}\left|\left(Q^{0}\right)^{t}\right| l_{H}^{\Lambda}\right\rangle+\frac{1}{(3 !)^{2}}\left(\frac{\partial^{3} V^{\Lambda}(\mathbf{R})}{\partial\left(Q^{\Lambda}\right)^{3}}\right)_{\mathbf{R}_{\Lambda}}^{2} \\
& \times \sum_{m, n \neq l}\left[\frac{\left\langle l_{H}^{\Lambda}\left|\left(Q^{\Lambda}\right)^{3}\right| m_{H}^{\Lambda}\right\rangle\left\langle m_{H}^{\Lambda}\left|\left(Q^{\Lambda}\right)^{3}\right| n_{H}^{\Lambda}\right\rangle\left\langle k_{H}^{0}\left|\left(Q^{0}\right)^{t}\right| n_{H}^{\Lambda}\right\rangle}{\hbar^{2}\left(\omega_{m \Lambda}^{H}-\omega_{l \Lambda}^{H}\right)\left(\omega_{n \Lambda}^{H}-\omega_{l \Lambda}^{H}\right)}\right]-\frac{1}{2(3 !)^{2}}\left(\frac{\partial^{3} V^{\Lambda}(\mathbf{R})}{\partial\left(Q^{\Lambda}\right)^{3}}\right)_{\mathbf{R}_{\Lambda}}^{2} \\
& \times \sum_{m \neq l}\left[\frac{\left\langle l_{H}^{\Lambda}\left|\left(Q^{\Lambda}\right)^{3}\right| m_{H}^{\Lambda}\right\rangle\left\langle m_{H}^{\Lambda}\left|\left(Q^{\Lambda}\right)^{3}\right| l_{H}^{\Lambda}\right\rangle}{\hbar^{2}\left(\omega_{m \Lambda}^{H}-\omega_{l \Lambda}^{H}\right)^{2}}\right]\left\langle k_{H}^{0}\left|\left(Q^{0}\right)^{t}\right| l_{H}^{\Lambda}\right\rangle+\cdots,
\end{aligned}
$$

where $t=0,1,2,3$ and, again, $Q^{0}$ and $Q^{\Lambda}$ represent the normal coordinates of the ground and $\Lambda$ state, respectively. Like expressions can be found for the integrals on the right of Eqs. (18) and (20).

We now have only to deal with integrals over harmonic oscillator functions, and for these we employ

$$
\begin{aligned}
\left\langle k_{H}^{0}\left|Q^{0}\right| l_{H}^{\Lambda}\right\rangle & =\left\langle l_{H}^{\Lambda}\left|\mathbf{Q}^{0}\right| k_{H}^{0}\right\rangle \\
& =\left\langle\phi_{l}^{\Lambda}\left|\mathbf{Q}^{0}\right| \phi_{k}^{0}\right\rangle \\
& =\left(\frac{\hbar}{2 \omega^{0}}\right)^{1 / 2}\left[\sqrt{k}\left\langle\phi_{l}^{\Lambda} \mid \phi_{k-1}^{0}\right\rangle+\sqrt{k+1}\left\langle\phi_{l}^{\Lambda} \mid \phi_{k+1}^{0}\right\rangle\right]
\end{aligned}
$$

and

$$
\left\langle\phi_{l}^{\Lambda}\left|\left(Q^{0}\right)^{t}\right| \phi_{k}^{0}\right\rangle=\sum_{j}\left\langle\phi_{l}^{\Lambda}\left|\left(Q^{0}\right)^{t-1}\right| \phi_{j}^{0}\right\rangle\left\langle\phi_{j}^{0}\left|Q^{0}\right| \phi_{k}^{0}\right\rangle ;
$$

here $t$ is an integer greater than unity, $\omega^{0}$ is the vibrational angular harmonic frequency of the electronic ground state and $k$ is the vibrational quantum number of the vibrational wavefunction $\phi_{k}^{0}$. Integrals of the type $\left\langle\phi_{j}^{0}\left|\left(Q^{0}\right)^{t}\right| \phi_{i}^{0}\right\rangle$ and $\left\langle\phi_{m}^{\Lambda}\left|\left(Q^{\Lambda}\right)^{t}\right| \phi_{n}^{\Lambda}\right\rangle$, which are also needed, can be found in the same way.

Finally the Franck-Condon integrals $\left\langle\phi_{j}^{0} \mid \phi_{m}^{\Lambda}\right\rangle$ for harmonic oscillator functions can be obtained from Eq. (2.9) of the paper by Iachello and Ibrahim. ${ }^{37}$ Through second-order in mechanical anharmonicity the vibrational energy differences in Eq. (10) are calculated, using the usual Dunham formula:

$$
\hbar \omega_{k 0}=k \hbar \omega_{e}\left[1-x_{e}(k+1)\right],
$$

where $\omega_{e}$ is the vibrational angular harmonic frequency of the electronic ground state and $\omega_{e} x_{e}$ is obtained from the cubic and quartic force constants in the conventional manner. Our results for the vibrational energies, see Table I, show that higher-order perturbation terms are negligible for the states considered.

\section{COMPUTATIONAL DETAILS}

We have chosen the carbon monoxide molecule for our initial implementation of the theory given in the preceding two sections. A major reason for this choice is that we may compare our results with those on the same molecule by Sundholm et al. ${ }^{27}$ They considered TPA for the $X^{1} \Sigma^{+}-A^{1} \Pi$ transition and took account of vibrational effects only as far as the vibrational averaging that occurs in the first term of Eq. (10). That is, the subsequent "pure" vibration terms in that equation were ignored.

Following them, we use the multiconfiguration self consistent field (MCSCF) ${ }^{40}$ method and response theory ${ }^{38,39}$ to construct our two potential energy curves $\left(X^{1} \Sigma^{+}, A^{1} \Pi\right),{ }^{41}$ the electronic ground state dipole moment function and the 
TABLE I. Vibrational energies $\hbar \omega_{k 0}$ (in a.u.) relative to the ground state for the $X^{1} \Sigma^{+}$electronic state of $\mathrm{CO}$.

\begin{tabular}{ccccc}
\hline \hline & $k=1$ & $k=2$ & $k=3$ & $k=4$ \\
\hline Harmonic $^{\mathrm{a}}$ & $1.003 \times 10^{-2}$ & $2.006 \times 10^{-2}$ & $3.008 \times 10^{-2}$ & $4.011 \times 10^{-2}$ \\
Anharmonic $^{\mathrm{a}}$ & $9.902 \times 10^{-3}$ & $1.968 \times 10^{-2}$ & $2.933 \times 10^{-2}$ & $3.886 \times 10^{-2}$ \\
Numeric & $9.915 \times 10^{-3}$ & $1.972 \times 10^{-2}$ & $2.941 \times 10^{-2}$ & $3.899 \times 10^{-2}$ \\
\hline \hline
\end{tabular}

${ }^{\mathrm{a} S e e ~ E q . ~(32) . ~}$

transition dipole moment ${ }^{41}$ and polarizability ${ }^{42,43}$ functions. The calculations were done with $C_{2 v}$ symmetry and the active orbitals in the complete active space (CAS) for the irreducible representations $A_{1}, B_{1}, B_{2}$ and $A_{2}$ were $4,2,2$ and 0 , respectively. The number of active electrons was 8 . The basis set used was Dunning's correlation consistent daug-ccpVTZ basis set. ${ }^{44,45}$ We carried out these computations with the DALTON program ${ }^{46}$ and confirmed our results by comparison with the values in Table VII of Ref. 27. Numerical differentiation of the energy and properties was carried out by the Romberg procedure. ${ }^{47}$

In using DALTON for obtaining the transition polarizability function we set in that program $\omega_{1}=\omega_{2}=\frac{1}{2} \omega_{\Lambda}$. Thus in Eqs. (6) and (7) we have $\omega=\frac{1}{2} \omega_{\Lambda}$ for each geometry $\mathbf{R}$ (N.B.: the optical frequency $\omega$ is now a function of $\mathbf{R}$ ). Necessarily, then, we are making an approximation here since in practical terms $\omega$ remains constant.

In this way we have all the $a b$ initio data necessary for getting the required integrals needed for the TPA crosssection of the $X^{1} \Sigma^{+}-A^{1} \Pi$ transition of $\mathrm{CO}$. We then can use the LEVEL 7.4 program $^{29}$ (benchmark) or, alternatively, perturbation theory to different orders. In all the calculations the experimental value of $\Gamma$ used, namely $1.39 \times 10^{-7} \mathrm{eV}$, corresponds to a lifetime of the $A^{1} \Pi$ state of $9.5 \mathrm{~ns}{ }^{48,49}$
TABLE II. Vibrational transition dipole moments, $\left\langle 0^{0}\left|\mu_{\alpha}^{00}(\mathbf{R})\right| k^{0}\right\rangle$, in a.u. for the electronic ground state of $\mathrm{CO} ; \alpha$ is the bond axis.

\begin{tabular}{lcccc}
\hline \hline & $k=1$ & $k=2$ & $k=3$ & $k=4$ \\
\hline$P(0)$ & $-4.262 \times 10^{-2}$ & 0.000 & 0.000 & 0.000 \\
$P(\mathrm{I})$ & $-4.262 \times 10^{-2}$ & $2.471 \times 10^{-3}$ & 0.000 & 0.000 \\
$P(\mathrm{II})$ & $-4.257 \times 10^{-2}$ & $2.471 \times 10^{-3}$ & $-1.328 \times 10^{-4}$ & 0.000 \\
$P(Z)^{\mathrm{a}}$ & $-4.256 \times 10^{-2}$ & $2.510 \times 10^{-3}$ & $-1.388 \times 10^{-4}$ & $-2.109 \times 10^{-5}$ \\
$P(\infty)^{\mathrm{b}}$ & $-4.257 \times 10^{-2}$ & $2.518 \times 10^{-3}$ & $-1.410 \times 10^{-4}$ & $5.641 \times 10^{-6}$ \\
\hline \hline
\end{tabular}

${ }^{\mathrm{a}} P(\mathrm{II})+P(1,2)+P(2,1)+P(2,2)$.

b،"Exact" numerical value.

\section{RESULTS AND DISCUSSION}

There are two objectives to this work: to study the role of nuclear vibrations in TPA and to determine the reliability of perturbation theoretical estimates of their importance. We consider the $X^{1} \Sigma^{+}-A^{1} \Pi$ transition in $\mathrm{CO}$ and deal with the reliability question first. The perturbation calculations can be done to various orders of electrical and mechanical anharmonicity and we can use the notation $(n, m)$ to denote the $n$th order of electrical and the $m$ th order of mechanical anharmonicity. If no anharmonicity is included, that is we use only the first two terms in the expansions in Eqs. (15)-(17), (21) and (22), we will call the results $P(0)$, i.e., only $(0,0)$ contributions. If we add the $(0,1)$ and $(1,0)$ terms, we label the result $P(\mathrm{I})$; and if we add to these $(0,2),(1,1)$ and $(2,0)$ we use the notation $P(\mathrm{II})$. We also make calculations which do not quite fit with this notation, namely those where we have taken all four terms in each of Eqs. (15)-(17), (21) and (22) (those through second-order in both types of anharmonicity), in other words $\mathrm{P}(\mathrm{II})+(2,1)+(1,2)+(2,2)$-we call these results $P(Z)$. The essentially exact results based on the vibra-

TABLE III. Vibrational transition dipole moments and polarizabilities between the electronic $X^{1} \Sigma^{+}$and $A^{1} \Pi$ states of $\mathrm{CO}$ (in a.u.). $\alpha$ is the bond axis and $\gamma$ is an axis perpendicular to $\alpha$.

\begin{tabular}{lccccc}
\hline \hline & $l=0$ & $l=1$ & $l=2$ & $l=3$ & $l=4$ \\
\hline$P(0)$ & & & & \\
$\left\langle 1^{0}\left|\mu_{\gamma}^{0 \Lambda}(\mathbf{R})\right| l^{\Lambda}\right\rangle$ & $2.32 \times 10^{-1}$ & $-2.17 \times 10^{-1}$ & $1.73 \times 10^{-2}$ & $2.13 \times 10^{-1}$ & $-3.30 \times 10^{-1}$ \\
$\left\langle 0^{0}\left|\alpha_{\alpha \gamma}^{0 \Lambda}(\mathbf{R}, \omega)\right| l^{\Lambda}\right\rangle$ & $-3.73 \times 10^{-2}$ & $7.53 \times 10^{-3}$ & $5.76 \times 10^{-2}$ & $-1.09 \times 10^{-1}$ & $1.18 \times 10^{-2}$ \\
$P(\mathrm{I})$ & & & & \\
$\left\langle 1^{0}\left|\mu_{\gamma}^{0 \Lambda}(\mathbf{R})\right| l^{\Lambda}\right\rangle$ & $2.57 \times 10^{-1}$ & $-2.36 \times 10^{-1}$ & $1.12 \times 10^{-1}$ & $3.75 \times 10^{-2}$ & $-1.94 \times 10^{-1}$ \\
$\left\langle 0^{0}\left|\alpha_{\alpha \gamma}^{0 \Lambda}(\mathbf{R}, \omega)\right| l^{\Lambda}\right\rangle$ & $-1.83 \times 10^{-2}$ & $7.84 \times 10^{-3}$ & $1.48 \times 10^{-2}$ & $-5.56 \times 10^{-2}$ & $1.09 \times 10^{-1}$ \\
$P(\mathrm{II})$ & & & & \\
$\left\langle 1^{0}\left|\mu_{\gamma}^{0 \Lambda}(\mathbf{R})\right| l^{\Lambda}\right\rangle$ & $2.59 \times 10^{-1}$ & $-2.26 \times 10^{-1}$ & $8.30 \times 10^{-2}$ & $6.01 \times 10^{-2}$ & $-1.44 \times 10^{-1}$ \\
$\left\langle 0^{0}\left|\alpha_{\alpha \gamma}^{0 \Lambda}(\mathbf{R}, \omega)\right| l^{\Lambda}\right\rangle$ & $-2.10 \times 10^{-2}$ & $4.92 \times 10^{-3}$ & $2.52 \times 10^{-2}$ & $-4.84 \times 10^{-2}$ & $6.18 \times 10^{-2}$ \\
$P(Z)^{\mathrm{a}}$ & & & & \\
$\left\langle 1^{0}\left|\mu_{\gamma}^{0 \Lambda}(\mathbf{R})\right| l^{\Lambda}\right\rangle$ & $2.59 \times 10^{-1}$ & $-2.26 \times 10^{-1}$ & $8.28 \times 10^{-2}$ & $6.03 \times 10^{-2}$ & $-1.44 \times 10^{-1}$ \\
$\left\langle 0^{0}\left|\alpha_{\alpha \gamma}^{0 \Lambda}(\mathbf{R}, \omega)\right| l^{\Lambda}\right\rangle$ & $-2.24 \times 10^{-2}$ & $5.34 \times 10^{-3}$ & $2.62 \times 10^{-2}$ & $-5.36 \times 10^{-2}$ & $6.77 \times 10^{-2}$ \\
$P(\infty)^{\mathrm{b}}$ & & & & \\
$\left\langle 1^{0}\left|\mu_{\gamma}^{0 \Lambda}(\mathbf{R})\right| l^{\Lambda}\right\rangle$ & $2.59 \times 10^{-1}$ & $-2.27 \times 10^{-1}$ & $8.51 \times 10^{-2}$ & $6.62 \times 10^{-2}$ & $-1.73 \times 10^{-1}$ \\
$\left\langle 0^{0}\left|\alpha_{\alpha \gamma}^{0 \Lambda}(\mathbf{R}, \omega)\right| l^{\Lambda}\right\rangle$ & $-2.11 \times 10^{-2}$ & $5.65 \times 10^{-3}$ & $2.57 \times 10^{-2}$ & $-5.73 \times 10^{-2}$ & $7.94 \times 10^{-2}$ \\
\hline \hline
\end{tabular}

asee Table II.

b“Exact" numerical value. 
TABLE IV. Orientationally averaged values (in a.u.) for the contributions to $\gamma^{\text {TP }}$, Eq. (13), and the TPA cross section, $\sigma^{\mathrm{TP}}$ (in $10^{-50} \mathrm{~cm}^{4} \mathrm{~s}$ /photon), Eq. (14), for two-photon energies in perfect resonance with the transition from the ground electronic and vibrational state to the lowest five vibrational levels of the $A^{1} \Pi(\Lambda)$ state.

\begin{tabular}{|c|c|c|c|c|c|}
\hline & $\omega=\frac{1}{2} \omega_{0 \Lambda}$ & $\omega=\frac{1}{2} \omega_{1 \Lambda}$ & $\omega=\frac{1}{2} \omega_{2 \Lambda}$ & $\omega=\frac{1}{2} \omega_{3 \Lambda}$ & $\omega=\frac{1}{2} \omega_{4 \Lambda}$ \\
\hline \multicolumn{6}{|l|}{$P(0)$} \\
\hline$\left\{\alpha^{2}\right\}^{\mathrm{TP}}$ & $4.36 \times 10^{6}$ & $1.76 \times 10^{5}$ & $1.04 \times 10^{7}$ & $3.75 \times 10^{7}$ & $4.37 \times 10^{7}$ \\
\hline$\left\{\mu^{2} \alpha\right\}^{\mathrm{TP}}$ & $-1.66 \times 10^{7}$ & $-3.04 \times 10^{6}$ & $1.82 \times 10^{6}$ & $-4.15 \times 10^{7}$ & $-6.79 \times 10^{7}$ \\
\hline$\left\{\mu^{4}\right\}^{\mathrm{TP}}$ & $1.57 \times 10^{7}$ & $1.32 \times 10^{7}$ & $7.97 \times 10^{4}$ & $1.15 \times 10^{7}$ & $2.63 \times 10^{7}$ \\
\hline$\gamma^{\mathrm{TP}}$ & $3.53 \times 10^{6}$ & $1.03 \times 10^{7}$ & $1.23 \times 10^{7}$ & $7.46 \times 10^{6}$ & $2.18 \times 10^{6}$ \\
\hline \multicolumn{6}{|l|}{$P(\mathrm{I})$} \\
\hline$\left\{\alpha^{2}\right\}^{\mathrm{TP}}$ & $1.11 \times 10^{6}$ & $2.11 \times 10^{5}$ & $6.80 \times 10^{5}$ & $9.66 \times 10^{6}$ & $3.71 \times 10^{7}$ \\
\hline$\left\{\mu^{2} \alpha\right\}^{\mathrm{TP}}$ & $-8.63 \times 10^{6}$ & $-3.54 \times 10^{6}$ & $3.24 \times 10^{6}$ & $-2.26 \times 10^{6}$ & $-3.43 \times 10^{7}$ \\
\hline$\left\{\mu^{4}\right\}^{\mathrm{TP}}$ & $1.68 \times 10^{7}$ & $1.49 \times 10^{7}$ & $3.85 \times 10^{6}$ & $1.33 \times 10^{5}$ & $7.94 \times 10^{6}$ \\
\hline$\gamma^{\mathrm{TP}}$ & $9.31 \times 10^{6}$ & $1.16 \times 10^{7}$ & $7.77 \times 10^{6}$ & $7.53 \times 10^{6}$ & $1.07 \times 10^{7}$ \\
\hline \multicolumn{6}{|l|}{$P(\mathrm{II})$} \\
\hline$\left\{\alpha^{2}\right\}^{\mathrm{TP}}$ & $1.31 \times 10^{6}$ & $6.49 \times 10^{4}$ & $2.01 \times 10^{6}$ & $7.32 \times 10^{6}$ & $1.19 \times 10^{7}$ \\
\hline$\left\{\mu^{2} \alpha\right\}^{\mathrm{TP}}$ & $-9.49 \times 10^{6}$ & $-1.89 \times 10^{6}$ & $4.20 \times 10^{6}$ & $-4.20 \times 10^{6}$ & $-1.46 \times 10^{7}$ \\
\hline$\left\{\mu^{4}\right\}^{\mathrm{TP}}$ & $1.72 \times 10^{7}$ & $1.37 \times 10^{7}$ & $2.19 \times 10^{6}$ & $6.04 \times 10^{5}$ & $4.49 \times 10^{6}$ \\
\hline$\gamma^{\mathrm{TP}}$ & $8.98 \times 10^{6}$ & $1.19 \times 10^{7}$ & $8.41 \times 10^{6}$ & $3.72 \times 10^{6}$ & $1.78 \times 10^{6}$ \\
\hline \multicolumn{6}{|l|}{$P(Z)^{\mathrm{a}}$} \\
\hline$\left\{\alpha^{2}\right\}^{\mathrm{TP}}$ & $1.50 \times 10^{6}$ & $7.74 \times 10^{4}$ & $2.18 \times 10^{6}$ & $8.99 \times 10^{6}$ & $1.43 \times 10^{7}$ \\
\hline$\left\{\mu^{2} \alpha\right\}^{\mathrm{TP}}$ & $-1.02 \times 10^{7}$ & $-2.06 \times 10^{6}$ & $4.37 \times 10^{6}$ & $-4.66 \times 10^{6}$ & $-1.61 \times 10^{7}$ \\
\hline$\left\{\mu^{4}\right\}^{\mathrm{TP}}$ & $1.72 \times 10^{7}$ & $1.37 \times 10^{7}$ & $2.19 \times 10^{6}$ & $6.04 \times 10^{5}$ & $4.51 \times 10^{6}$ \\
\hline$\gamma^{\mathrm{TP}}$ & $8.51 \times 10^{6}$ & $1.17 \times 10^{7}$ & $8.74 \times 10^{6}$ & $4.94 \times 10^{6}$ & $2.76 \times 10^{6}$ \\
\hline \multicolumn{6}{|l|}{$P(\infty)^{\mathrm{b}}$} \\
\hline$\left\{\alpha^{2}\right\}^{\mathrm{TP}}$ & $1.40 \times 10^{6}$ & $1.00 \times 10^{5}$ & $2.06 \times 10^{6}$ & $1.02 \times 10^{7}$ & $1.97 \times 10^{7}$ \\
\hline$\left\{\mu^{2} \alpha\right\}^{\mathrm{TP}}$ & $-9.78 \times 10^{6}$ & $-2.35 \times 10^{6}$ & $4.36 \times 10^{6}$ & $-5.50 \times 10^{6}$ & $-2.28 \times 10^{7}$ \\
\hline$\left\{\mu^{4}\right\}^{\mathrm{TP}}$ & $1.71 \times 10^{7}$ & $1.38 \times 10^{7}$ & $2.31 \times 10^{6}$ & $7.37 \times 10^{5}$ & $6.62 \times 10^{6}$ \\
\hline$\gamma^{\mathrm{TP}}$ & $8.74 \times 10^{6}$ & $1.15 \times 10^{7}$ & $8.73 \times 10^{6}$ & $5.49 \times 10^{6}$ & $3.50 \times 10^{6}$ \\
\hline$\sigma^{\mathrm{TP}}$ & $1.63 \times 10^{2}$ & $2.25 \times 10^{2}$ & $1.78 \times 10^{2}$ & $1.17 \times 10^{2}$ & $7.75 \times 10^{1}$ \\
\hline
\end{tabular}

${ }^{\mathrm{a}}$ See Table II

b،"Exact" numerical value.

tional wavefunctions determined by the program LEVEL $7.4,^{29}$ followed by numerical integration, will be labeled by $\mathrm{P}(\infty)$.

In Table I we show the vibrational energies of the four lowest excited vibrational states (relative to the ground state) for the $X^{1} \Sigma^{+}$state of CO. We do so at the harmonic level and anharmonic level [Eq. (32)], and we compare these numbers with the "exact" numerical values. It is clear that our anharmonic values, through second-order, agree with the exact numerical values to within $0.3 \%$.

In Table II values of the vibrational transition dipole moments $\left\langle 0^{0}\left|\mu_{\alpha}^{00}(\mathbf{R})\right| k^{0}\right\rangle$ are shown, where the superscript 0 indicates the $X^{1} \Sigma^{+}$ground electronic state of $\mathrm{CO}$, and, of course, the dipole transition moments lie along the internuclear axis. Values are given for the vibrational levels with $k$ $=1,2,3$ and 4 , however for the last two cases the transition moment is negligible. For $k=1$ and $k=2$ there is nice convergence from $P(\mathrm{I})$ through $P(\mathrm{II}), P(Z)$ to $P(\infty)$; the last two cases giving nearly identical results. The $P($ II) values appear therefore to be satisfactory with relative errors compared to $P(\infty)$ smaller than $2 \%$.

In Table III we present values of the transition dipole and polarizability between the electronic ground state $(0$ $\left.=X^{1} \Sigma^{+}\right)$and the excited state $\left(\Lambda=A^{1} \Pi\right)$ using the first vibrational level of the ground state for the dipole and the zero level for the polarizability. These are the important levels in the evaluation of $\gamma^{\mathrm{TP}}$. Numbers are given for the lowest five vibrational states of the $\Lambda$ electronic state, i.e., $l$ $=0,1,2,3$ and 4 . The levels of perturbation approximation are $P(0), P(\mathrm{I}), P(\mathrm{II})$ and $P(Z)$ and comparison is made to the exact values given by $P(\infty)$. In general, the convergence with regard to increasing higher levels of perturbation theory is very good. The maximum errors relative to $P(\infty)$ are $222 \%, 43 \%, 22 \%$ and $17 \%$ for $P(0), P(\mathrm{I}), P(\mathrm{II})$ and $P(Z)$, respectively.

TABLE V. "Exact" values (in a.u.) of transition dipole moments and polarizabilities between the $X^{1} \Sigma^{+}$ground electronic state and a fictitious $A^{1} \Pi(\Lambda)$ excited state with the same curvature, but with the equilibrium geometry of the true $A{ }^{1} \Pi(\Lambda)$ state of CO. $\alpha$ is the bond axis and $\gamma$ is an axis perpendicular to $\alpha$.

\begin{tabular}{cccccc}
\hline \hline & $l=0$ & $l=1$ & $l=2$ & $l=3$ & $l=4$ \\
\hline$\left\langle 1^{0}\left|\mu_{\gamma}^{0 \Lambda}(\mathbf{R})\right| l^{\Lambda}\right\rangle$ & $2.53 \times 10^{-1}$ & $-2.41 \times 10^{-1}$ & $1.45 \times 10^{-1}$ & $-3.05 \times 10^{-2}$ & $-6.62 \times 10^{-2}$ \\
$\left\langle 0^{0}\left|\alpha_{\alpha \gamma}^{0 \Lambda}(\mathbf{R}, \omega)\right| l^{\Lambda}\right\rangle$ & $-2.48 \times 10^{-2}$ & $1.68 \times 10^{-2}$ & $4.80 \times 10^{-3}$ & $-2.96 \times 10^{-2}$ & $5.08 \times 10^{-2}$ \\
\hline \hline
\end{tabular}


TABLE VI. "Exact" orientationally averaged values for the contributions to $\gamma^{\mathrm{TP}}$, Eq. (13), at two-photon energies coincident with the lowest vibrational levels of a fictitious excited state with the same curvature as the ground state $X^{1} \Sigma^{+}$but with the equilibrium geometry of the true $A^{1} \Pi(\Lambda)$ state, in a.u.

\begin{tabular}{cccccc}
\hline \hline & $\omega=\frac{1}{2} \omega_{0 \Lambda}$ & $\omega=\frac{1}{2} \omega_{1 \Lambda}$ & $\omega=\frac{1}{2} \omega_{2 \Lambda}$ & $\omega=\frac{1}{2} \omega_{3 \Lambda}$ & $\omega=\frac{1}{2} \omega_{4 \Lambda}$ \\
\hline$\left\{\alpha^{2}\right\}^{\mathrm{TP}}$ & $1.92 \times 10^{6}$ & $8.82 \times 10^{5}$ & $7.15 \times 10^{4}$ & $2.73 \times 10^{6}$ & $8.07 \times 10^{6}$ \\
$\left\{\mu^{2} \alpha\right\}^{\mathrm{TP}}$ & $-1.11 \times 10^{7}$ & $-7.26 \times 10^{6}$ & $1.28 \times 10^{6}$ & $2.12 \times 10^{6}$ & $-4.70 \times 10^{6}$ \\
$\left\{\mu^{4}\right\}^{\mathrm{TP}}$ & $1.60 \times 10^{7}$ & $1.49 \times 10^{7}$ & $5.70 \times 10^{6}$ & $4.11 \times 10^{5}$ & $6.82 \times 10^{5}$ \\
$\gamma^{\mathrm{TP}}$ & $6.82 \times 10^{6}$ & $8.55 \times 10^{6}$ & $7.05 \times 10^{6}$ & $5.26 \times 10^{6}$ & $4.06 \times 10^{6}$ \\
\hline \hline
\end{tabular}

Table IV incorporates the most significant quantities to be presented in this work, that is, the contributions to $\gamma^{\mathrm{TP}}$ as given by Eq. (10). Five levels of theory (including the "exact") are presented. The levels of theory here refer to transition dipoles and polarizabilities such as those given in Tables II and III. Second-order contributions to the vibrational energy denominators are included only at second-order and higher levels of theory, i.e., $P(\mathrm{II})$ and $P(Z)$. Results are shown for the five two-photon frequencies which coincide with the first five vibrational levels of the $A^{1} \Pi$ state of $\mathrm{CO}$ (starting from the vibrational ground state of $X^{1} \Sigma^{+}$). There is, in general, good convergence as more perturbation terms are included. For the first three optical frequencies, the $P$ (II) and $P(Z)$ results for $\gamma^{\mathrm{TP}}$ agree with $P(\infty)$ to within $3.7 \%$ and $2.6 \%$, respectively. Bigger discrepancies are found for other optical frequencies; the worst case is $\omega=\frac{1}{2} \omega_{4 \Lambda}$ with a relative error for $\gamma^{\mathrm{TP}}$ with respect to $P(\infty)$ of $49 \%$ and $21 \%$ for $P(\mathrm{II})$ and $P(Z)$, respectively. The total "exact" TPA cross-sections $\sigma^{\mathrm{TP}}$, see Eq. (14), are given in the last row.

With Table IV at hand we address the key question of the importance of vibration in TPA. To do so, we focus on the $P(\infty)$, or exact results, at the bottom of the table. The pure electronic contribution (the complete neglect of vibration) to $\gamma^{\mathrm{TP}}$ given by Eq. (12) is of the form $\left\{\alpha^{2}\right\}$. It has the value $4.07 \times 10^{7}$ a.u. for $\omega=1 / 2 \omega_{\Lambda}$ and $\mathbf{R}=\mathbf{R}_{\mathbf{0}}$ and is approximately twice as big as the largest of the $\left\{\alpha^{2}\right\}^{\mathrm{TP}}$ values given in the $P(\infty)$ section of Table IV. Further calculations (not presented) at optical frequencies in resonance with higher vibrational states of the electronic state $\Lambda$ lead to the same conclusion. Furthermore the "pure" vibrational contributions $\left\{\mu^{2} \alpha\right\}^{\mathrm{TP}}$ and $\left\{\mu^{4}\right\}^{\mathrm{TP}}$ are larger in magnitude than $\left\{\alpha^{2}\right\}^{\mathrm{TP}}$, though they are of opposite sign and, to an extent, cancel each other for some of the optical frequencies. Nonetheless, we witness a large change in $\gamma^{\mathrm{TP}}$ with the addition of the "pure" vibrational contributions to the $\left\{\alpha^{2}\right\}^{\mathrm{TP}}$ term for all five optical frequencies.

Finally, we consider the legitimacy of assuming that the excited state PES of CO is of the same shape as the ground state but simply shifted to the correct equilibrium position. This is an assumption frequently made in the literature for other molecules; see, e.g., Ref. 25. The calculations reported in Tables V and VI are all carried out at the $P(\infty)$ level. A comparison of the transition integrals in Table $\mathrm{V}$ with those in Table III shows that, except for $l=0$, this is a very crude approximation. The maximum errors relative to $P(\infty)$ in Table III are $146 \%$ and $197 \%$ for $\left\langle 1^{0}\left|\mu_{\gamma}^{0 \Lambda}(\mathbf{R})\right| l^{\Lambda}\right\rangle$ and $\left\langle 0^{0}\left|\alpha_{\alpha \gamma}^{0 \Lambda}(\mathbf{R}, \omega)\right| l^{\Lambda}\right\rangle$, respectively. In like fashion, a comparison of Tables VI and IV shows that, except for the optical frequency $\omega=1 / 2 \omega_{0 \Lambda}$, the assumption of the same shape for the ground and excited PES can be a fair approximation for calculating $\gamma^{\mathrm{TP}}$ but it is a procedure not to be generally recommended.

This is not the first time that the vibrational effects in TPA have been investigated, but all previous attempts have been much less complete. In nearly all cases attention has zeroed in on the $\left\{\alpha^{2}\right\}^{\mathrm{TP}}$ term where vibrational averaging has been done exactly by Sundholm et $a l^{27}$ or approximately by Ågren and co-workers ${ }^{24-26}$ (either just the first or the first two terms in the expansion of Eq. (17) have been included). Hence in these calculations the "pure" vibrational terms $\left\{\mu^{2} \alpha\right\}^{\mathrm{TP}}$ and $\left\{\mu^{4}\right\}^{\mathrm{TP}}$ were excluded. In addition, the investigations of Agren and co-workers were made without mechanical anharmonicity. It is sometimes assumed that the ground and excited PES have identical shape ${ }^{25}$ which, as we have shown, can lead to substantial errors. It may also be noted that in Ref. 26 the vibrational subscript $l$, that occurs in the leading square bracket in Eq. (10), is ignored and closure is made over the vibrational states of the excited electronic state. In this work we carry out the summation over $l$ correctly. Quite recently Painelli et $a l^{28}$ have alluded to the "pure" vibrational contributions to TPA but in a quite different and less rigorous framework-their calculations were based on a crude two-state model (for a critique of this model see Ref. 50) and purely harmonic potential energy surfaces.

In summary, we have laid out a rigorous and transparent treatment of the effects of nuclear vibrational motion in TPA. We have developed practical perturbation formulas for the case of diatomic molecules and applied these formulas to the $X^{1} \Sigma^{+}-A^{1} \Pi$ transition in $\mathrm{CO}$. We find that vibration can play an important role in TPA, just as it does in the calculation of conventional NLO hyperpolarizabilities. Attention is drawn to the "pure" vibrational terms $\left\{\mu^{2} \alpha\right\}^{\mathrm{TP}}$ and $\left\{\mu^{4}\right\}^{\mathrm{TP}}$, which turn out to be particularly important for $\mathrm{CO}$. We also see that both mechanical and electrical anharmonicities can be important. Our next concern is the generalization and application of the theory to polyatomic molecules.

\section{ACKNOWLEDGMENTS}

D.M.B and J.M.L. thank the Natural Sciences and Engineering Research Council of Canada for funding. J.M.L. acknowledges financial support for this work under Grant No. PB98-0457-C02-01 from the Dirección General de Enseñanza Superior e Investigación Científica y Técnica (MEC-Spain). 
${ }^{1}$ D. M. Bishop and B. Kirtman, J. Chem. Phys. 95, 2646 (1991).

${ }^{2}$ D. M. Bishop, J. M. Luis, and B. Kirtman, J. Chem. Phys. 108, 10013 (1998).

${ }^{3}$ D. M. Bishop, Adv. Chem. Phys. 104, 1 (1998).

${ }^{4}$ D. M. Bishop, Rev. Mod. Phys. 62, 343 (1990).

${ }^{5}$ B. Kirtman and B. Champagne, Int. Rev. Phys. Chem. 16, 389 (1997).

${ }^{6}$ M. Albota, D. Beljonne, J. L. Brédas et al., Science 281, 1653 (1998).

${ }^{7}$ P. Callis, Annu. Rev. Phys. Chem. 48, 271 (1997).

${ }^{8}$ A. A. Said, C. Wamsley, D. J. Hagan, E. W. Van Stryland, B. A. Reinhart,

P. Roderer, and A. G. Dillard, Chem. Phys. Lett. 228, 646 (1994).

${ }^{9}$ J. E. Ehrlich, X. L. Wu, I.-Y. S. Lee, Z.-Y. Hu, H. Rockel, S. R. Marder, and J. W. Perry, Opt. Lett. 22, 1843 (1997).

${ }^{10}$ J. H. Strickler and W. W. Webb, Opt. Lett. 16, 1780 (1991).

${ }^{11}$ W. Deuk, Proc. Natl. Acad. Sci. U.S.A. 91, 6629 (1997).

${ }^{12}$ J. D. Bhawalkar, G. S. He, and P. N. Prasad, Rep. Prog. Phys. 59, 1041 (1996).

${ }^{13}$ B. A. Reinhardt, L. L. Brott, S. J. Clarson, A. G. Dillard, J. C. Bhatt, R. Kannan, L. Yuan, G. S. He, and P. N. Prasad, Chem. Mater. 10, 1863 (1998).

${ }^{14}$ G. I. Stegeman, SPIE Proc. 1852, 75 (1993).

${ }^{15}$ P. Norman, D. M. Bishop, H. J. Aa. Jensen, and J. Oddershede, J. Chem. Phys. 115, 10323 (2001).

${ }^{16}$ Y. Luo, H. Ågren, and S. Stafström, J. Phys. Chem. 98, 7782 (1994).

${ }^{17}$ Y. Luo, O. Vahtras, and H. Ågren, Chem. Phys. Lett. 204, 587 (1993)

${ }^{18}$ P. Norman, Y. Luo, and H. Ågren, Chem. Phys. Lett. 296, 8 (1998).

${ }^{19}$ P. Norman, Y. Luo, and H. Ågren, J. Chem. Phys. 111, 7758 (1999).

${ }^{20}$ K. Kogej, D. Beljonne, F. Meyers, J. W. Perry, S. R. Marder, and J. L. Brédas, Chem. Phys. Lett. 228, 1 (1998).

${ }^{21}$ B. Honig, J. Jortner, and A. Szoke, J. Chem. Phys. 46, 2714 (1967).

${ }^{22}$ B. Honig and J. Jortner, J. Chem. Phys. 47, 3698 (1967).

${ }^{23}$ Y. Luo, H. Ågren, S. Knuts, B. F. Minaev, and P. Jørgensen, Chem. Phys. Lett. 209, 513 (1993).

${ }^{24}$ Y. Luo, H. Ågren, S. Knuts, and P. Jørgensen, Chem. Phys. Lett. 213, 356 (1993).

${ }^{25}$ P. Macak, Y. Luo, P. Norman, and H. Ågren, J. Chem. Phys. 113, 7055 (2000).

${ }^{26}$ P. Macak, Y. Luo, P. Norman, and H. Ågren, Chem. Phys. Lett. 330, 447 (2000).

${ }^{27}$ D. Sundholm, J. Olsen, and P. Jørgensen, J. Chem. Phys. 102, 4143 (1995).
${ }^{28}$ A. Painelli, L. Del Freo, and F. Terenziani, Chem. Phys. Lett. 346, 470 (2001).

${ }^{29}$ R. J. Le Roy, LEVEL 7.4. A computer program for solving the radial Schrödinger equation for bound and quasibound levels, University of Waterloo Chemical Physics Research Report CP-642 R ${ }^{3}, 2001$.

${ }^{30}$ B. J. Orr and J. F. Ward, Mol. Phys. 20, 513 (1971).

${ }^{31}$ P. N. Butcher and D. Cotter, The Elements of Nonlinear Optics (Cambridge University Press, Cambridge, 1993).

${ }^{32}$ A. D. Buckingham and P. Fischer, Phys. Rev. A 61, 035801 (2000).

${ }^{33}$ D.M. Bishop, L. M. Cheung, and A. D. Buckingham, Mol. Phys. 41, 1225 (1980).

${ }^{34}$ D. M. Bishop, B. Kirtman, and B. Champagne, J. Chem. Phys. 107, 5780 (1997).

${ }^{35}$ D. M. Bishop, J. Chem. Phys. 100, 6535 (1994).

${ }^{36}$ J. O. Hirschfelder, W. Byers Brown, and S. T. Epstein, Adv. Quantum Chem. 1, 255 (1964).

${ }^{37}$ F. Iachello and M. Ibrahim, J. Phys. Chem. A 102, 9427 (1998).

${ }^{38}$ D. L. Yeager and P. Jørgensen, Chem. Phys. Lett. 65, 77 (1979).

${ }^{39}$ J. Olsen and P. Jørgensen, J. Chem. Phys. 82, 3235 (1985).

${ }^{40}$ H. J. Aa. Jensen and H. Ågren, Chem. Phys. Lett. 104, 229 (1986).

${ }^{41}$ P. Jørgensen, H. J. Aa. Jensen, and J. Olsen, J. Chem. Phys. 89, 3654 (1988).

${ }^{42}$ H. Hettema, H. J. Aa. Jensen, P. Jørgensen, and J. Olsen, J. Chem. Phys. 97, 1174 (1992).

${ }^{43}$ O. Vahtras, H. Ågren, P. Jørgensen, H. J. Aa. Jensen, T. Helgaker, and J. Olsen, J. Chem. Phys. 96, 6120 (1992).

${ }^{44}$ T. H. Dunning, Jr., J. Chem. Phys. 90, 1007 (1989).

${ }^{45}$ R.A. Kendall, T. H. Dunning, Jr., and R. J. Harrison, J. Chem. Phys. 96, 6796 (1992)

${ }^{46}$ DALTON, a molecular electronic structure program, Release 1.2, 2001, written by T. Helgaker, H. J. Aa. Jensen, P. Jørgensen et al.

${ }^{47}$ P. J. Davis and P. Rabinowitz, Numerical Integration (Blaisdell, London, 1967), p. 166.

${ }^{48}$ A. C. Le Floch, F. Launay, J. Rostas, R. W. Field, C. M. Brown, and K. Yoshino, J. Mol. Spectrosc. 121, 337 (1987).

${ }^{49}$ T. Sycora and C. R. Vidal, J. Chem. Phys. 108, 6320 (1998).

${ }^{50}$ D. M. Bishop, B. Champagne, and B. Kirtman, J. Chem. Phys. 109, 9987 (1998). 